\title{
Etude et prototypage d'un générateur de signaux arbitraires à vocation didactique
}

\author{
J. MBIHI ${ }^{(*)}$, N. TENE et K. TAKOUFET \\ Groupe de Recherche en Informatique Industrielle et Automation (GR2IA) \\ Ecole Normale Supérieure d'Enseignement Technique (ENSET), B.P. 1872, Douala, Cameroun.
}

(*) E-mail : mbihidr@yahoo.fr

\section{Résumé}

L'instrument programmable considéré dans cet article est un générateur de signaux arbitraires à but didactique contrôlé par ordinateur. Le logiciel de contrôle dudit instrument permet de simuler et de générer par ordinateur une grande variété de signaux arbitraires via une interface matérielle propriétaire ou commerciale. Les principes de conception et les techniques de mise en œuvre de cet instrument sont dévoilés. Puis un prototype d'instrument virtuel réalisé est décrit et les échantillons de signaux arbitraires obtenus sont présentés.

Mots clés : Instrument programmable, signaux arbitraires, logiciel de contrôle, interface matérielle, prototype d'instrument virtuel.

\section{Introduction}

Un générateur de signaux arbitraires (GSA) est un instrument programmable, conçu pour délivrer une grande variété de signaux permettant de tester des circuits et systèmes électroniques [1]. Depuis plusieurs années, cette classe d'instruments programmable ne cesse d'attirer l'attention des fabricants de produits d'appoint en instrumentation. Les exemples de modèles de GSA commerciaux sont: AFG-320 de Tektronics, AWGU2761A de Acquitek, AWS-8770A de Hewlett Packard, K8016 de Velleman, Iqwave-802.11 de LitePoint Corporation, TGA-1240 de Thurlby Thandar Instruments et NI-5412 de National Instrument. Ces variétés de produits commerciaux cités sont des instruments sophistiqués, performants et coûteux. Ils sont généralement constitués d'un microsystème matériel à processeur de signaux embarqué et réglable par ordinateur (figure 1).

Malgré l'intérêt grandissant de GSA commerciaux, les enseignements techniques permettant aux apprenants de génie électrique de maîtriser leur conception et construction, sont rares sinon quasiment inexistants dans les programmes de formation en génie électrique. Par ailleurs, ce type d'enseignement ne serait efficace que si le laboratoire de formation des apprenants dispose de prototypes de GSA dont les secrets de construction (documentation technique, codes sources et exécutables du logiciel de contrôle) sont dévoilés. Cependant, ces besoins et exigences pédagogiques ne constituent pas la priorité des instruments commerciaux.

Le but de cet article est de présenter les principes de conception ainsi que les techniques de mise en œuvre d'un GSA à but didactique. Dans la section 2, nous présenterons le principe de fonctionnement d'un GSA. Les sections 3 et 4 porteront respectivement sur l'architecture matérielle et la mise en œuvre logicielle d'un prototype d'instrument. Puis, les caractéristiques techniques dudit instrument seront présentées dans la section 5 . Enfin, les échantillons de signaux arbitraires produits seront présentés à la section 6.

\section{Principe de fonctionnement du GSA}

Le principe d'un GSA consiste à simuler numériquement des signaux par ordinateur, puis à générer les signaux obtenus via une 


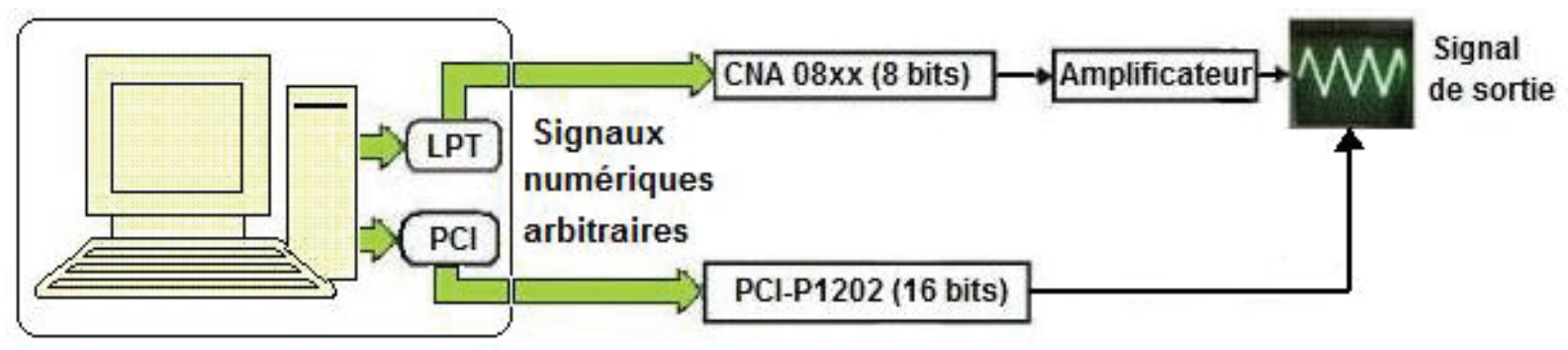

Fig. 1 : Prototype de GSA proposé

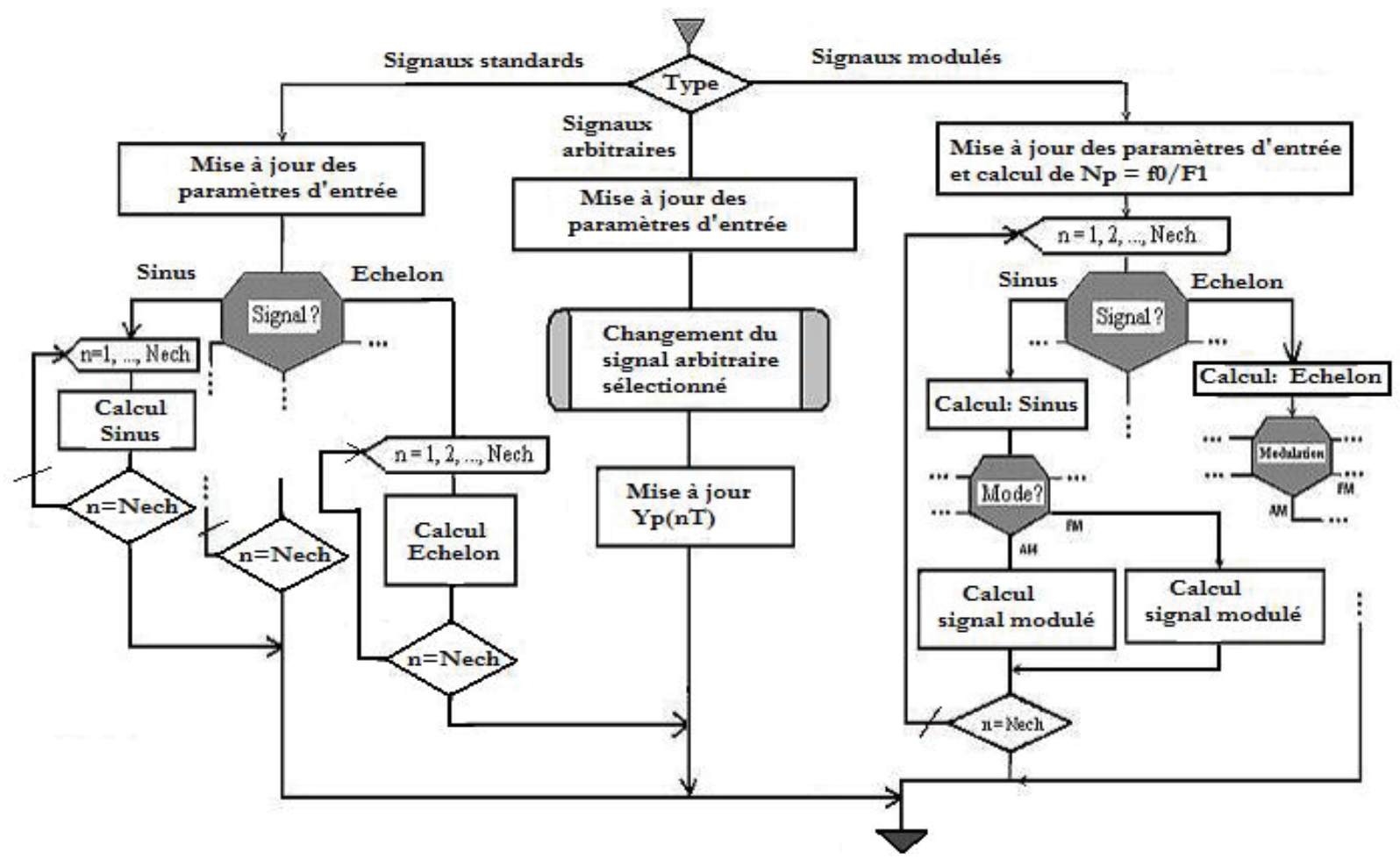

Fig. 2: Schéma algorithmique de calcul numérique des signaux

interface matérielle de conversion analogique-numérique. Ainsi, les algorithmes de simulation numérique de signaux arbitraires, sont implémentés et exécutés du côté ordinateur, ce qui entraîne une sollicitation importante du processeur.

Le prototype de GSA à but didactique proposé à la figure 1 , a été construit au laboratoire de recherche en informatique industrielle et automation de l'ENSET de Douala, dans le cadre des projets de fin d'études [2-3]. L'étude et l'expérimentation de cet instrument permettent aux étudiants de niveau Masters en IIA (Informatique Industrielle et Automation) et EEA (Electronique, Electrotechnique et Automatique) de l'ENSET de Douala de :

a) comprendre les principes et techniques de conception et de mise en œuvre des GSA programmables par ordinateur, étudiées dans un chapitre de l'unité d'enseignement d'instrumentation et asservissement par ordinateur.

c) tester des circuits électroniques à l'aide d'une source de tension arbitraire réglable par ordinateur.

\section{Architecture matérielle du GSA}

La partie matérielle du GSA proposé est un système combiné d'interfaces de conversion numérique-analogique (N/A) pour port LPT ('Line printer') et pour bus d'extension PCI (Peripheral Component Interconnect). 
Dans le premier cas, plusieurs modèles d'interfaces LPT du GSA ont été construits dans le cadre des travaux de mémoires de fin d'études [2-3] de niveau Master.

Dans le second cas, le convertisseur N/A est un chip bipolaire de 16 bits embarqué dans le modèle de carte d'interface PCI-P1202 de ICP DAS [4]. Le driver P1202.DLL fournit avec cette carte facilite l'accès aux adresses matérielles sous Windows 2000/XP, et permet en outre de générer la période d'échantillonnage à l'aide d'un Timer externe.

\section{Mise en œuvre logicielle du GSA}

Le schéma algorithmique de calcul numérique des signaux correspond à la figure 2. Pour générer numériquement un signal standard (simple ou modulé) périodique $Y p(n)$ de fréquence $F_{1}$, connaissant $N p$ premières valeurs par période $\left\{\mathrm{Y}_{\mathrm{p}}(1), \mathrm{Y}_{\mathrm{p}}(2), \ldots, \mathrm{Y}_{\mathrm{p}}(\mathrm{Np})\right\}$, les valeurs futures $Y_{p}(N p+1), Y_{p}(N p+2), \ldots$, sont calculées en appliquant la propriété de périodicité qui se traduit dans ce cas par la relation :

$$
Y_{p}(n)=Y_{p}\left(n-N_{p}\right) \quad \forall n>N_{p}
$$

En supposant que $f_{0}$ soit la fréquence d'échantillonnage utilisée, alors le nombre $N p$ requis correspond à :

$$
N_{p}=\text { Partie entière de } \frac{f_{0}}{F_{1}}
$$

où $F_{1}$ est la fréquence maximale contenue dans le spectre de $Y_{\mathrm{p}}(\mathrm{n})$ et $f_{0}>2 F$ d'après le théorème d'échantillonnage.

Dans le cas d'un signal arbitraire, les valeurs sont pré-calculées et chargées en mémoire en temps d'exécution à partir d'une base de données enregistrée sur disque.

La procédure d'événement permettant de générer en temps réel un signal préalablement simulé et visualisé, correspond au schéma algorithmique représenté à la figure 3. La boucle de génération est contrôlée par un Timer de

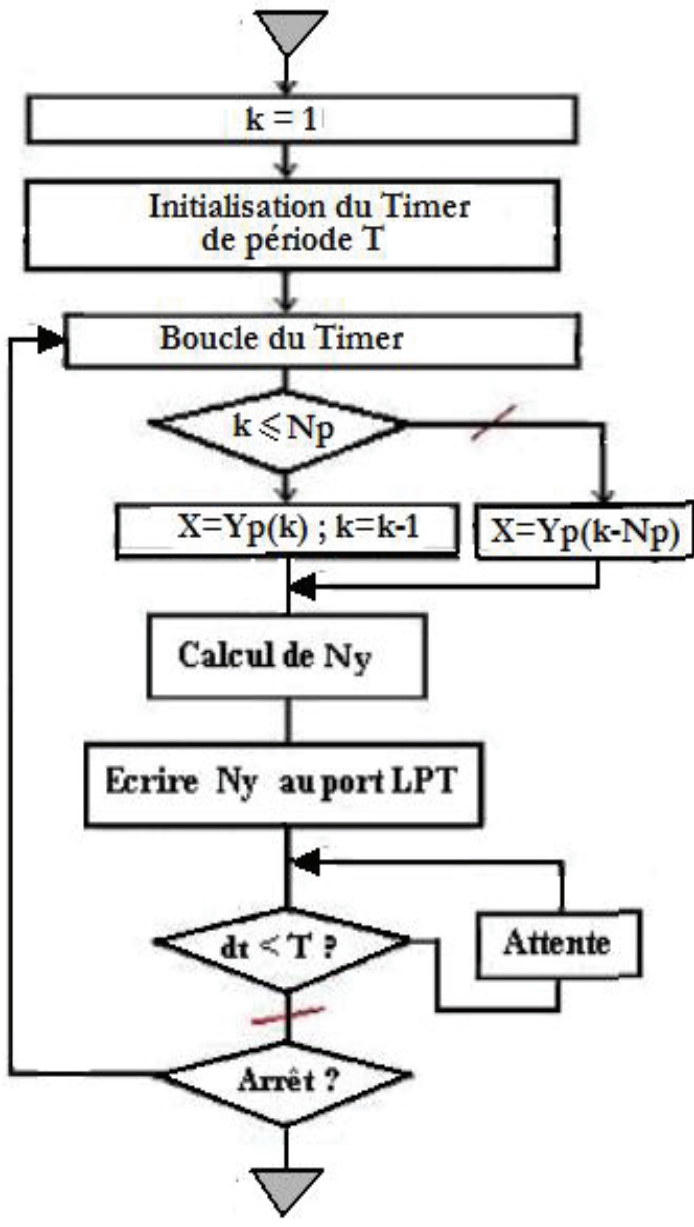

Fig. 3 : Génération en temps réel d'un signal

période T. Dans un cycle de génération, la valeur actualisée du signal en cours est codée, puis appliquée au bus de données de l'interface de conversion sélectionné.

Le logiciel de contrôle du prototype du GSA, a été développé en Visual Basic conformément aux schémas algorithmiques précédemment définis (figures 2 et 3 ) et suivant les techniques de programmation système détaillés dans [5]. Ce logiciel est une outil d'instrumentation virtuelle dont le panneau de contrôle offre les possibilités suivantes :

a) Deux langues d'affichage.

b) Deux types d'interface : LPC et PCI.

c) Types de signaux multiples.

d) Paramètres de signaux réglables.

e) Visualisation des résultats.

\section{Caractéristiques techniques du GSA}

La famille d'interfaces LPT-DAC-08xx de 8 bits est conçu pour fonctionner dans la plage 
\pm 12 Volts. Les performances qui en résultent sont les suivantes :

- Résolution : $\left(24 / 2^{8}\right)=94 \mathrm{mV}$,

- Bande passante : $30 \mathrm{Khz}$ à raison de 10 échantillons par période pour un débit de 300 Kilo-octet/s.

- Courant de sortie $: \pm 150 \mathrm{~mA}$.

Par ailleurs, l'interface PCI-P1202 de 12 bits est réglée pour fonctionner dans la plage \pm 10 Volts. Elle admet pour caractéristiques techniques [4] :

- Résolution : $\left(20 / 2^{12}\right)=19,5 \mathrm{mV}$,

- Bande passante : $400 \mathrm{Khz}$ à raison de
10 échantillons par période pour un débit de $4 \mathrm{Mo} / \mathrm{s}$.

- Courant de sortie : $\pm 5 \mathrm{~mW}$.

Ces caractéristiques sont suffisantes pour le test de la plupart de circuits électroniques à but didactique.

\section{Essais du GSA proposé}

Dans l'environnement d'essais du prototype de GSA proposé, les bornes de sortie d'une interface cible sont reliées à l'entrée de l'instrument de mesure Hewlett Packard HP1631D. La figure 4 correspond au

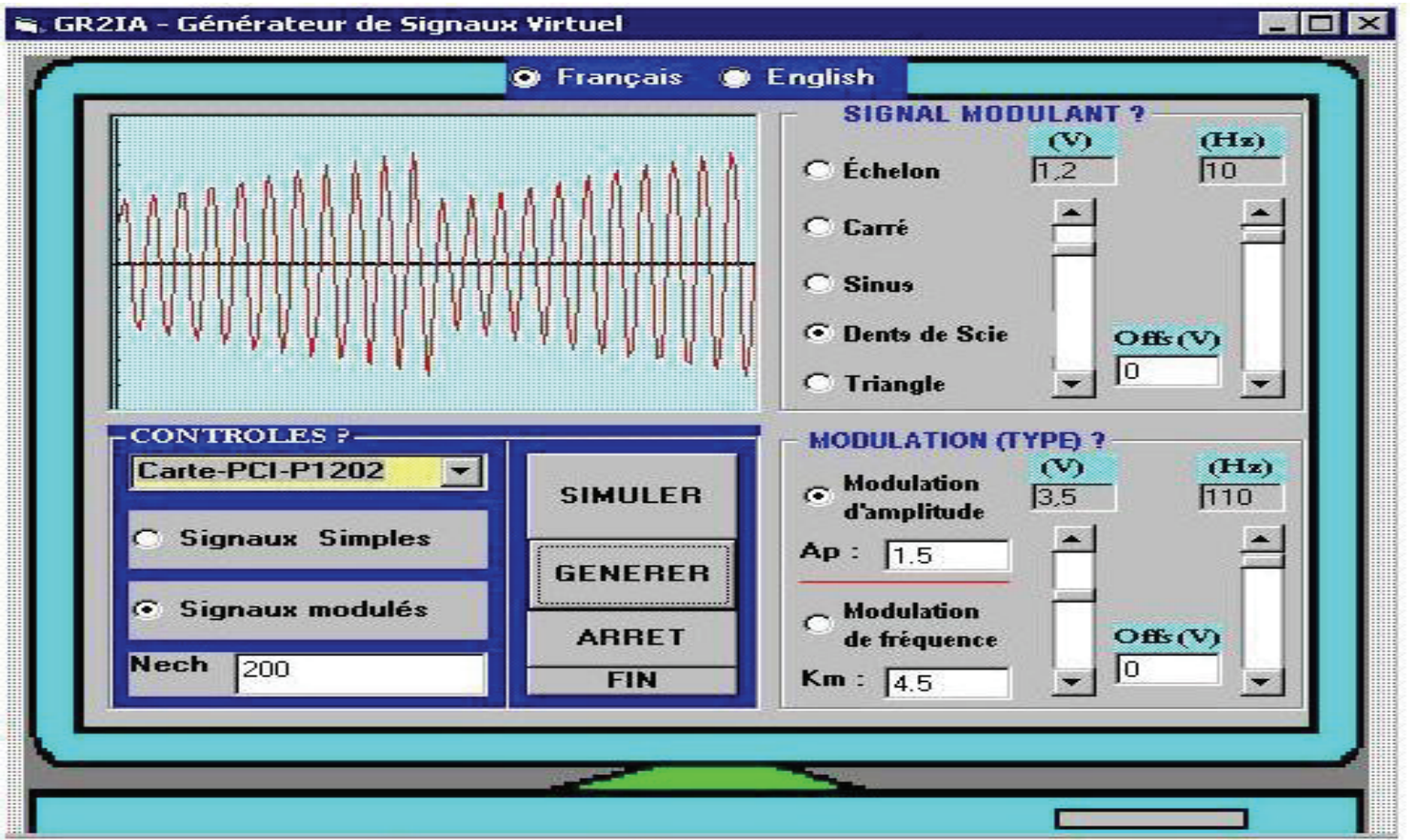

a) Panneau de contrôle virtuelle

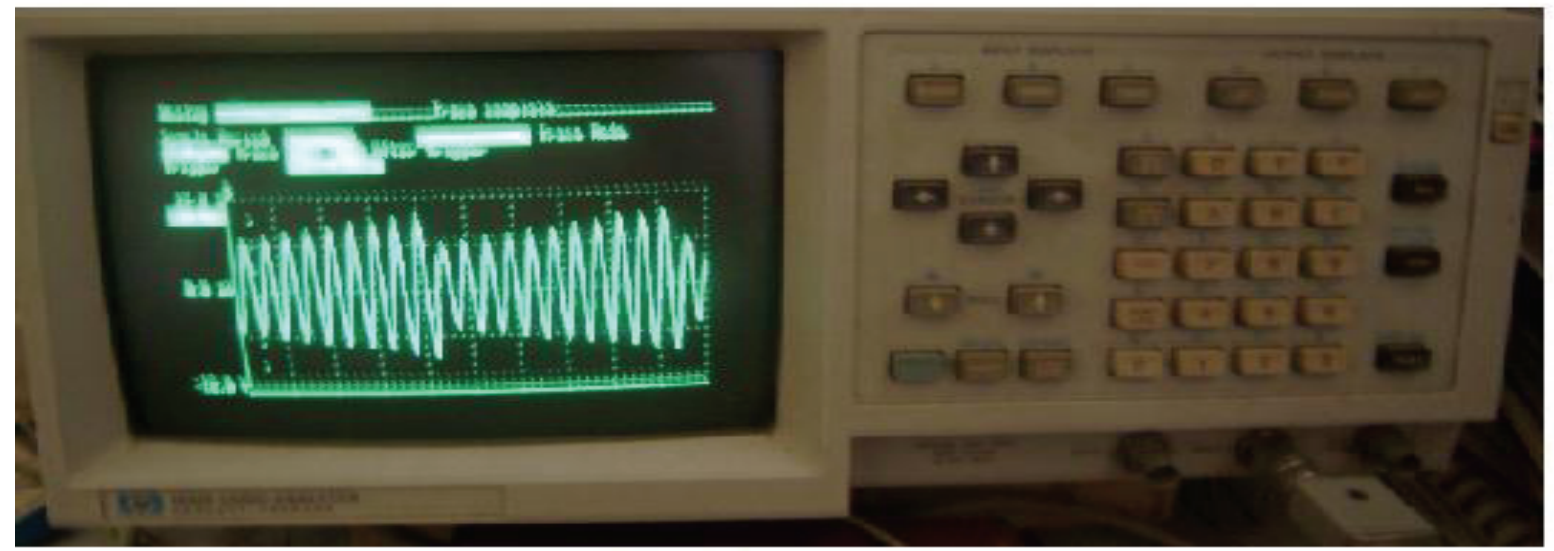

b) Face avant de l'instrument de mesure

Fig. 4 : Génération du signal sinusoïdal modulé en AM via la carte d'interface PCI-P1202 
résultat de génération d'un signal en dents de scie modulé en amplitude, via la carte d'interface PCI-P1202. Les échantillons de résultats d'essais obtenus pour d'autres types de signaux standards sont résumés dans la figure 5, où seuls les écrans d'affichage des instruments sont montrés pour des raisons de clarté. La figure 5(a) correspond au cas de la génération de trois exemples de signaux simples (carré, triangle, sinus). Par ailleurs, la figure 5(b) montre deux exemples de génération de signaux (carrés et triangulaire) modulés en amplitude (AM), ainsi qu'un exemple de génération de signal sinusoïdal modulé en fréquence (FM). Enfin, les exemples de signaux arbitraires générés sont présentés à la figure 6 . Le premier signal arbitraire (figure 6(a)) est constitué d'une alternance de signal sinusoïdal simple et de son image modulée en amplitude, alors que le $2^{\mathrm{e}}$ signal arbitraire (figure. 6(b)) associé à l'option ArbSource2 est une ondelette qui résulte de la troncature d'un signal sinusoïdal AM.

La qualité des résultats d'essais présentés ont permis de valider les principes de conception et les techniques de mise en œuvre, du modèle de GSA à vocation didactique présenté dans cet article. Nos futurs travaux de recherche porteront sur l'amélioration des performances de cet instrument.

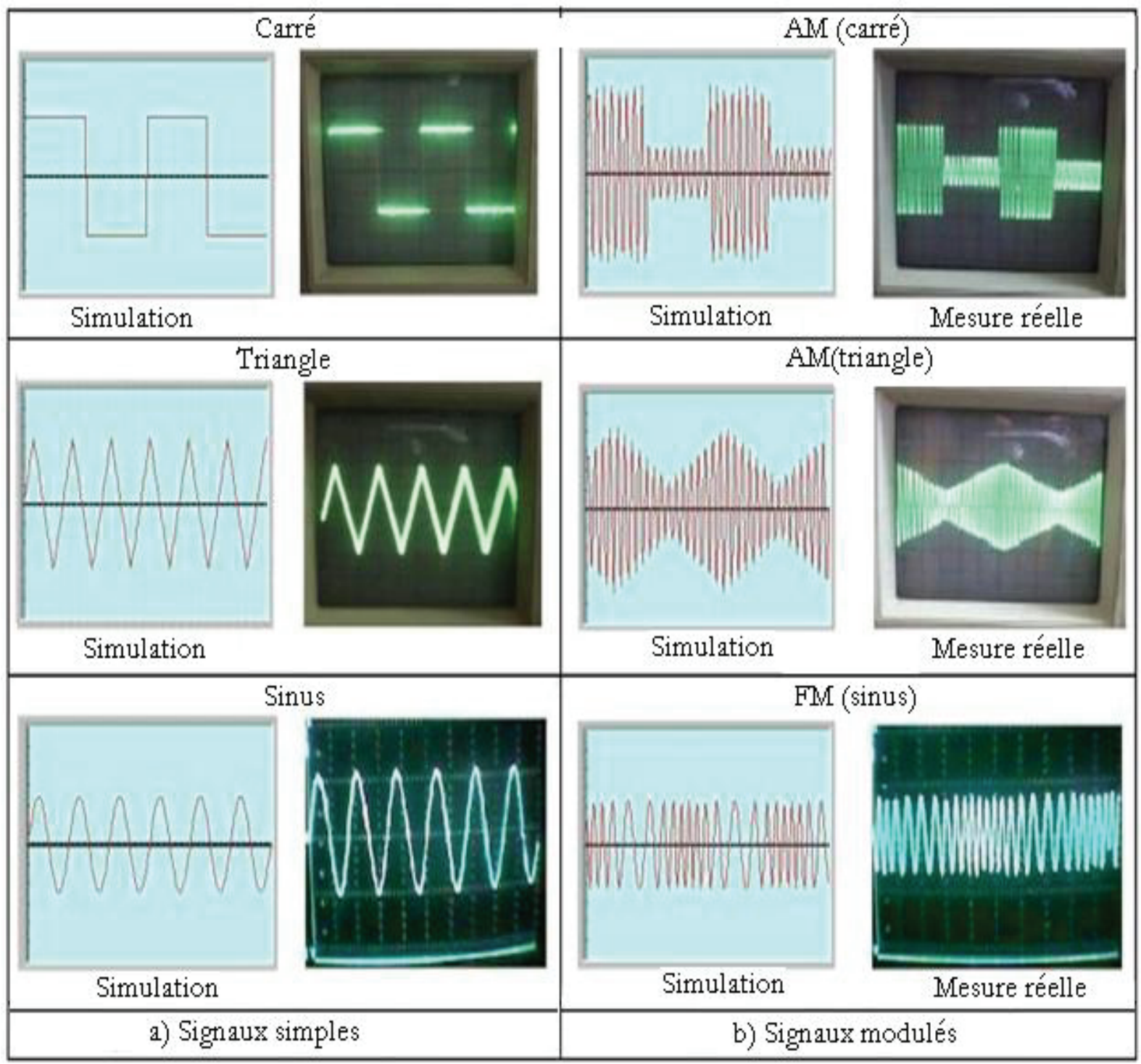

Fig. 5: Autres types de signaux générés 


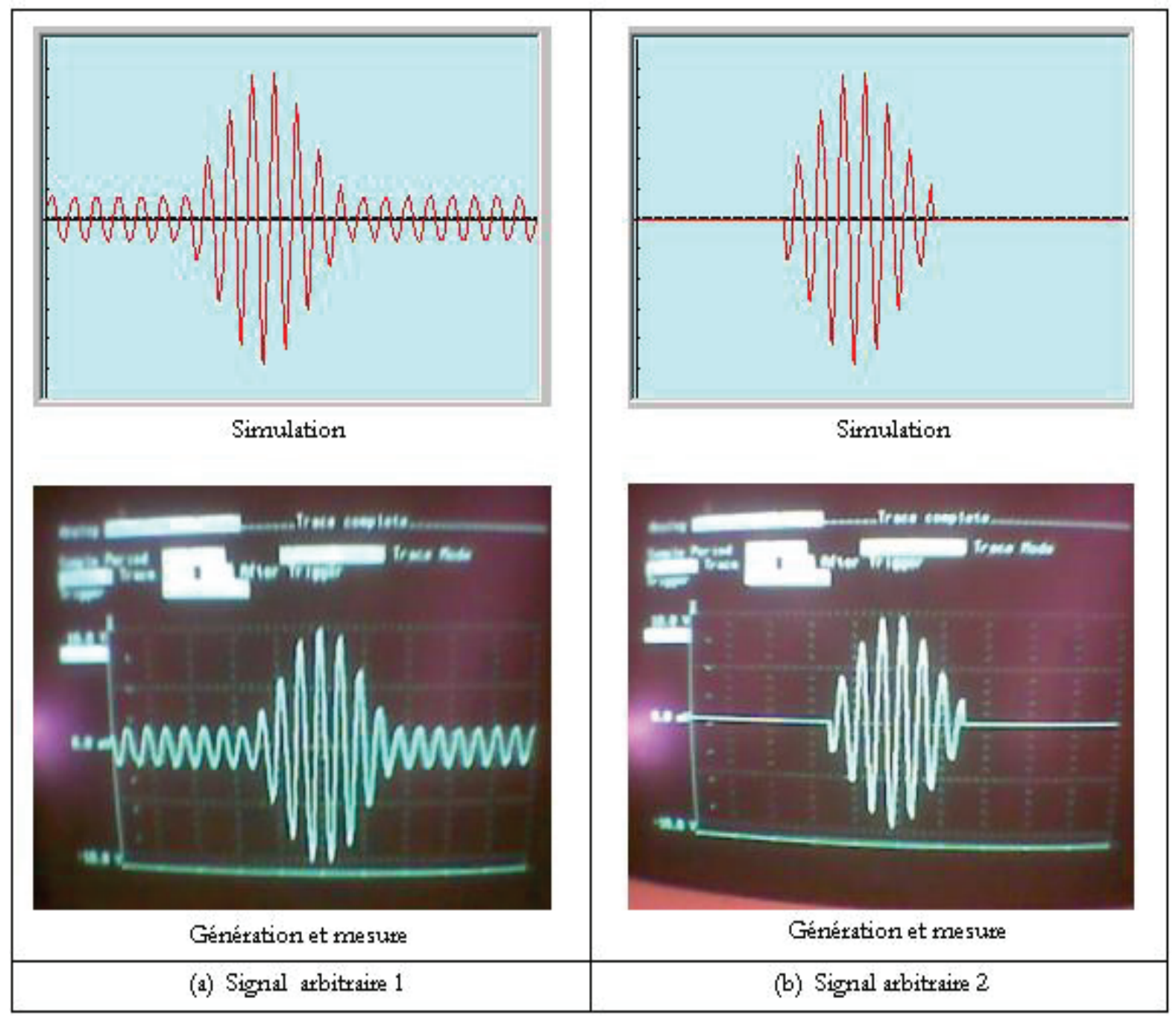

Fig. 6: Résultats de simulation et de génération en temps réel des signaux arbitraires

\section{Conclusion}

L'instrument programmable présenté dans cet article est un matériel didactique d'appoint, utilisé dans le cadre de l'unité d'enseignement d'instrumentation et de commande par ordinateur, destinée aux étudiants de Master en IIA et EEA de l'ENSET de Douala. La disponibilité des documents descriptifs détaillés et du code source Visual Basic du logiciel de contrôle, facilitent un apprentissage approfondi des secrets de conception et de mise en œuvre, ce qui n'est pas possible dans le cas des instruments commerciaux.

Une issue d'extension de ses possibilités est un éditeur d'équations embarqué pour signaux arbitraires définis analytiquement. Il serait aussi intéressant d'envisager la mie en œuvre d'un module d'interface de génération de signaux via le port USB de l'ordinateur.

\section{Références bibliographiques}

[1] National Instrument. "Measurement and Automation Catalogue: Arbitrary Waveform generators Overview and Tutorial”. 1999.

[2] NZOUANKE, "Conception et réalisation d'un Générateur de fonctions programmable par ordinateur". Mémoire de fin d'études en vue de l'obtention du DIPET II, GR2IA-GEL, ENSET, Université de Douala, Cameroun, 2005. 
[3] N. TENE et K. TAKOUFET, "Conception et réalisation d'un générateur de signaux modulés par ordinateur". Mémoire de fin d'études en vue de l'obtention du DIPET II, GR2IA-GEL, ENSET, Université de Douala, Cameroun, 2007.

[4] ICP DAS, “PCI- P1202/1602/1800/1802 Hardware User's manual - Version 3.4”, 2003, ICP DAS.

[5] J. MBIHI, "Informatique et automation: Automatismes programmables contrôlés par ordinateur", Editions Publibook, 2006, Paris.

\section{Remerciements}

Les auteurs tiennent à remercier sincèrement les rapporteurs anonymes de J3EA qui ont examiné en profondeur le manuscrit de cet article. Leurs suggestions et remarques très constructives, ont permis d'améliorer le contenu de la version finale de l'article.

\section{Biographie des auteurs}

J. MBIHI a obtenu le Ph. D. (1999) et le MSc.A. (1992) en génie électrique à l'École Polytechnique de Montréal (Québec, Canada). Il est actuellement Maitre de conférence, responsable du Groupe de Recherche en Informatique Industrielle et Automation (GR2IA), et chef du département d'Industrie Textile et de l'Habillement à l'ENSET de Douala (Cameroun). Il est membre du comité scientifique de l'unité de formation doctorale en Physique et Sciences de l'Ingénieur de l'Université de Douala, et membre du Comité Consultatif des Institutions Universitaires du Cameroun. Il est auteur de l'ouvrage "Informatique et Automation: Automatismes programmables contrôlés par ordinateur", publié aux éditions Publibook de Paris en 2006. Ses recherches actuelles portent sur les nouvelles technologies d'instrumentation virtuelle et de commande par ordinateur des processus à distance via Internet.

$N$. TENE et $K$. TAKOUFET sont titulaires du diplôme de Professeur d'Enseignement
Technique (DIPET) de $2^{\mathrm{e}}$ grade en EEA, obtenu à l'ENSET de Douala au Cameroun en 2007. Dans le cadre de leur mémoire de fin d'études pour l'obtention du DIPET de 2e grade, ils ont participé au sein du Groupe de Recherche en Informatique Industrielle et Automation (GR2IA) aux travaux de construction du prototype d'interface matérielle LPT-DAC0808 décrit dans cet article. 